\title{
The role of topical Genta Fleece HD and gentamicin spray in prevention of sternum wound infections after open heart surgery: a comparative study
}

Mazlum Sahin

Haseki Education and Research Hospital Cardiovascular Surgery, Istanbul, Turkey

Submitted: 27 January 2018

Accepted: 28 January 2018

Arch Med Sci Atheroscler Dis 2018; 3: e29-e34

DOI: https://doi.org/10.5114/amsad.2018.73526

Copyright $\odot 2018$ Termedia \& Banach

\begin{abstract}
Introduction: The aim was to clarify and compare the efficacy of local Genta Fleece HD (GF) and topical gentamicin spray (GS) in prevention of wound infections by comparing their results with a control group.

Material and methods: In this retrospective study, charts of patients who underwent coronary artery bypass grafting in a tertiary academic center between January 2015 and January 2017 were analyzed. Patients were divided into three groups: patients in whom GF was applied, patients in whom GS was applied, and a control group in which no additional material was applied. All patients received intravenous antibiotic prophylaxis. Deep and superficial sternal wound infections (SWIs) during the postoperative period were analyzed.

Results: The overall infection rate was $13.8 \%(n=51)$. Patients in whom GF was applied had significantly fewer SWIs when compared to the GS group and control group $(p=0.002)$. A significantly smaller number of deep SWIs occurred in the GS group $(7 / 177 ; 3.9 \%)$ than in the control group $(14 / 125$; $11.2 \%)$. The superficial SWI rate was significantly lower $(2 / 79 ; 2.5 \%)$ in the GF group than in the other two groups. Duration of hospital stay was significantly shorter in the GF group, followed by the GS and control groups. High EuroSCORE results $(\geq 4)$, obesity $\left(\mathrm{BMI}>30 \mathrm{~kg} / \mathrm{m}^{2}\right)$, performing the operations under emergency situations and presence of chronic obstructive pulmonary disease were identified as significant risk factors in the development of SWI. Conclusions: Local GS reduced deep SWIs, whereas GF implantation reduced the overall and superficial SWIs. Also, GF application was superior in terms of reducing the duration of hospital stay.
\end{abstract}

Key words: mediastinitis, complication, gentamicin, sternal wound infection, local antibiotic.

\section{Introduction}

Sternal wound infections (SWIs) are serious postoperative complications that increase the morbidity and mortality of heart surgery. Routine intravenous antibiotic prophylaxis has been shown to reduce the rates of postoperative infections and their associated complications [1]. Local application of gentamicin on the incision area before wound closure was shown to provide a higher concentration of the antibiotic at the surgical site without reaching systemic toxic levels [2].

The literature provides controversial evidence regarding the use of topical antibiotics. Application of gentamicin collagen sponge was shown

\author{
Corresponding author: \\ Mazlum Sahin \\ Haseki Education and \\ Research Hospital \\ Cardiovascular Surgery \\ 34250 Istanbul, Turkey \\ Phone: +90 5346194374 \\ E-mail: mzlmshn@gmail.com
}


to reduce the rates of SWIs in three meta-analysis [3-5], whereas another meta-analysis failed to demonstrate benefits of gentamicin collagen sponge usage [6]. Some authors sprayed a mixture of cefazolin and gentamicin antibiotics on the wound and they stated that this technique reduces deep sternal wound infections (DSWIs) $[7,8]$.

The objective of this study is to clarify and compare the efficacy of local Genta Fleece HD application (GF) and topical gentamicin spray (GS) at the surgical wound by comparing their results with a control group.

\section{Material and methods}

In this retrospective study, charts of patients who underwent coronary artery bypass grafting (CABG) in a tertiary academic center between January 2015 and January 2017 were analyzed. All patients underwent isolated CABG with a median sternotomy incision. Patients were divided into three groups according to the type of local antibiotic application at the end of the operation. Patients were divided into three groups: patients in whom GF was applied, patients in whom GS was applied and a control group in which no additional material was applied. All patients were given a routine prophylactic antibiotic regimen with cefazolin sodium, which was approved by the institutional department of infectious diseases. Standard surgical wound care was applied to all patients. Patients' demographic data, medical history and risk factors for wound infection were

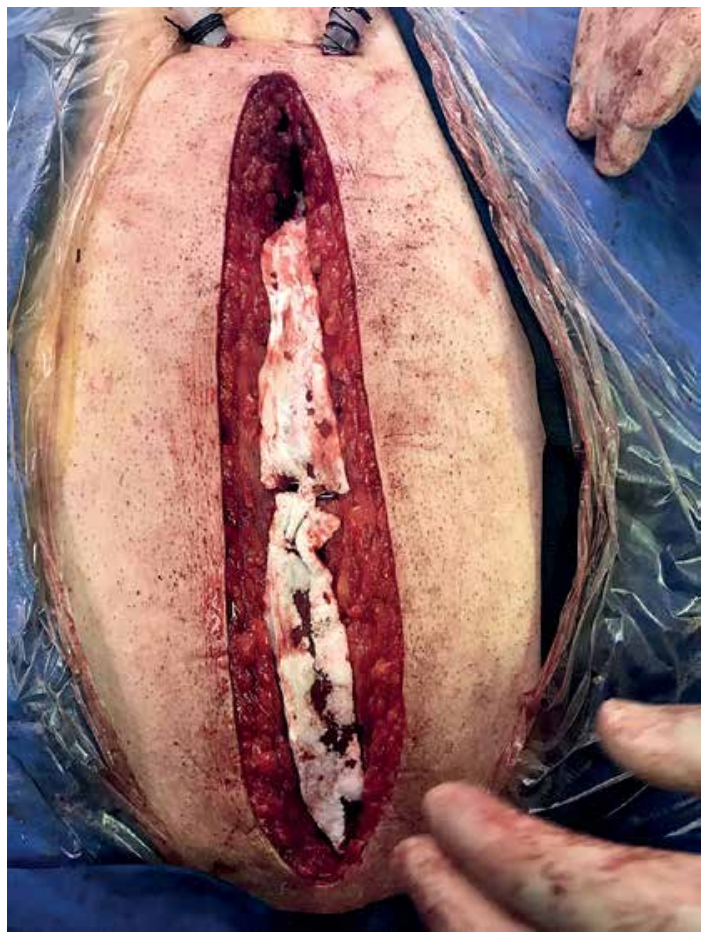

Figure 1. Application of the Genta Fleece HD collected. Also, operative and postoperative parameters were recorded.

Surgical wounds were inspected daily for signs of infection during the postoperative period. The patients were routinely followed at the outpatient clinic at the first week, first month and 2 months after discharge from the hospital. All patients were followed up to a period of 2 months after surgery. The patients were instructed to re-visit the clinic if they noticed any sign of infection.

Classification of deep or superficial surgical site infection was done according to the criteria suggested by the Centers of Disease Control [9]. Superficial infections were considered as infections involving the cutis and/or the subcutaneous tissues but not reaching the sternal fixation wires. Deep surgical infections were considered as infections reaching posterior to the fascia, involving the sternal wires, unstable sternal fixation with signs of osteomyelitis or positive bacterial cultures from the mediastinum or the presence of a mediastinal abscess.

\section{Surgical technique}

According to our pre-operative protocol, the skin at surgical sites was scrubbed with chlorhexidine $4 \%$ solution before the operation. After induction of general anesthesia, the skin was shaved and scrubbed with alcoholic iodine solution. Antibiotic prophylaxis was initiated $30 \mathrm{~min}$ before the first incision. One gram of cefazolin was given every $6^{\text {th }} \mathrm{h}$ and it was continued for at least $24 \mathrm{~h}$.

Surgery was performed in a strict sterile surgical environment. At the end of surgery, the sternum was fixed using 6 to 8 wires. The modified Robicsek technique or sternal plaques were used in certain cases. The fascia, subcutaneous tissues and skin were sutured using running absorbable sutures. No staples were used. Post-operatively, all patients were transported to the cardiac surgical intensive care unit where they were extubated following our institutional protocol.

\section{Application of the Fleece Genta HD}

Strips of Parasorb Fleece Genta HD (AMS Group, Winsford, Cheshire, U.K.) were allowed to absorb a solution containing $25 \mathrm{mg}$ of gentamicin sulfate. These strips were placed above the sternal wires after fixation of the sternum as shown in Figure 1. The subcutaneous tissues and skin were closed as describe above.

\section{Application of gentamicin spray}

A $10 \mathrm{ml}$ solution containing $80 \mathrm{mg}$ of gentamicin was prepared and it was sprayed on the wound after the sternal wires were placed. The 
subcutaneous tissues and the skin were closed as described above.

\section{Statistical analysis}

Descriptive statistics were used to report patients' demographic data. Data were expressed as mean \pm standard deviation for continuous variables and percentages for categorical variables. The Mann-Whitney $U$ test was used to compare the continuous variables between the three study groups. The $\chi^{2}$ test was used to evaluate categorical variables. $P$-values $<0.05$ were considered statistically significant. Sub-group analysis was performed using the Bonferroni correction whenever the above-mentioned tests showed significant results. A $p$-value < 0.017 was considered significant in sub-group analysis. All patients who died or who were re-operated on for post-operative bleeding were included in the analysis. Patients who were transferred to the inten- sive care unit with an open sternum for persisting bleeding were not included in the study. Risk factors for sternal wound infections were identified using univariable and multivariable logistic regression analysis. The associations of independent predictors with SWI were expressed as odds ratios (OR) with 95\% confidence intervals (Cls). All statistical analyses were performed using SPSS version 22.0 (IBM-SPSS Inc, Armonk, NY).

\section{Results}

From January 2015 to January 2017, 381 patients who underwent CABG in a tertiary academic center were enrolled in the study. Obesity, diabetes mellitus and chronic obstructive pulmonary disease were significantly more common among patients who have had post-operative infection ( $p=0.011, p<0.001$ and $p=0.016$, respectively). Also, EuroSCORE was higher in patients in whom

Table I. Comparison of patients according to the presence of postoperative wound infection

\begin{tabular}{|c|c|c|c|}
\hline Parameter & Infection $(n=51)$ & No infection $(n=333)$ & $P$-value \\
\hline Closure method: & & & 0.001 \\
\hline Group 1 & $26(51.0 \%)$ & 99 (29.7\%) & \\
\hline Group 2 & $21(41.2 \%)$ & $159(47.8 \%)$ & \\
\hline Group 3 & $4(7.8 \%)$ & $75(22.5 \%)$ & \\
\hline Gender (male/female) & $36 / 15$ & $250 / 83$ & 0.428 \\
\hline Age [year]* & $64.9 \pm 8.9$ & $65.5 \pm 8.5$ & 0.608 \\
\hline Body mass index $\left[\mathrm{kg} / \mathrm{m}^{2}\right]^{*}$ & $31.2 \pm 3.2$ & $29.9 \pm 3.5$ & 0.011 \\
\hline \multicolumn{4}{|l|}{ Comorbidity: } \\
\hline Diabetes mellitus & $39(76.5 \%)$ & $120(36.0 \%)$ & $<0.001$ \\
\hline Hypertension & $17(33.3 \%)$ & $158(47.4 \%)$ & 0.060 \\
\hline Chronic renal failure & $6(11.8 \%)$ & $23(6.9 \%)$ & 0.223 \\
\hline Chronic obstructive pulmonary disease & $16(31.4 \%)$ & $57(17.1 \%)$ & 0.016 \\
\hline Cancer & $6(11.8 \%)$ & $18(5.4 \%)$ & 0.081 \\
\hline Smoking history & $19(37.3 \%)$ & $147(44.1 \%)$ & 0.356 \\
\hline EuroSCORE* & $4.1 \pm 1.3$ & $2.9 \pm 1.5$ & $<0.001$ \\
\hline Cardiac ejection fraction* & $47.1 \pm 10.8$ & $48.8 \pm 9.9$ & 0.235 \\
\hline Operation under emergency conditions & $8(15.7 \%)$ & $7(2.1 \%)$ & $<0.001$ \\
\hline CABG & $3.3 \pm 0.9$ & $3.0 \pm 0.8$ & 0.023 \\
\hline Blood transfusion & $2.4 \pm 1.1$ & $1.3 \pm 0.7$ & $<0.001$ \\
\hline Revision due to hemorrhage & $10(19.6 \%)$ & $14(4.2 \%)$ & $<0.001$ \\
\hline Intensive care unit time $[\text { day }]^{*}$ & $5.3 \pm 5.5$ & $2.2 \pm 0.4$ & $<0.001$ \\
\hline Hospitalization time $[\text { day }]^{\star}$ & $13.3 \pm 5.7$ & $5.2 \pm 0.6$ & $<0.001$ \\
\hline Mortality & $5(9.8 \%)$ & $9(2.7 \%)$ & 0.012 \\
\hline
\end{tabular}

${ }^{\star}$ Mean \pm standard deviation. CABG - coronary artery bypass grafting. 
Table II. Comparison of infection rates and bacterial culture results among groups

\begin{tabular}{|c|c|c|c|c|}
\hline Parameter & Control group & $\begin{array}{c}\text { Gentamicin spray } \\
\text { group }\end{array}$ & $\begin{array}{l}\text { Topical Genta } \\
\text { Fleece HD }\end{array}$ & $P$-value \\
\hline All infections (\%): & $26(20.8)$ & $21(11.6)$ & $4(5.0)$ & 0.004 \\
\hline Superficial SWI & $12(9.5)$ & $14(7.7)$ & $2(2.5)$ & 0.009 \\
\hline Deep SWI & $14(11.1)$ & $7(3.9)$ & $2(2.5)$ & 0.011 \\
\hline Positive bacterial cultures (\%): & $19(73.1)$ & $13(61.9)$ & $3(75.0)$ & 0.325 \\
\hline MRSA & $4(15.4)$ & $8(38.1)$ & $2(50.0)$ & \\
\hline MSSA & $9(34.6)$ & $3(14.3)$ & $0(0.0)$ & \\
\hline Escherichia coli (\%) & $3(11.5)$ & $1(4.8)$ & $1(25.0)$ & \\
\hline Enterobacter (\%) & $2(7.7)$ & $1(4.8)$ & $0(0.0)$ & \\
\hline Pseudomonas (\%) & $1(3.8)$ & $0(0.0)$ & $0(0.0)$ & \\
\hline
\end{tabular}

SWI - sternal wound infections, MRSA - methicillin-resistant Staphylococcus aureus, MSSA - methicillin-sensitive Staphylococcus aureus.

infectious complications had occurred $(p<0.001)$ (Table I).

Among the 381 patients included in the study, $51(13.8 \%)$ cases of SWI occurred post-operatively. In total, a significantly smaller proportion of SWIs occurred in the GF group $(4 / 79 ; 5.1 \%, p=0.002)$ compared to the control and GS groups. Additionally, 21/177 (11.9\%) of the patients in the GS group had SWI compared to $26 / 125$ (20.8\%) in the control group, but this difference was not statistically significant. Evaluation of superficial (SSWIs) vs. deep SWIs (DSWIs) revealed a significantly lower number of SSWIs $(2 / 79 ; 2.5 \%)$ in the GF group than in the other two groups. A significantly smaller number of DSWIs occurred in the GS group $(7 / 177 ; 3.9 \%)$ than in the control group (14/125; 11.2\%) (Table II).

Evaluation of duration of hospital stay showed a significantly shorter stay in the GF group (5.3 \pm 1.6 days, $p<0.01$ ). Application of GS was also associated with a significantly shorter hospital stay (6.2 \pm 3.6 days, $p<0.001)$ compared to the control group $(7.1 \pm 4.0$ days; $p<0.001)$. Hospital stay was found to be shorter in the GF group than in the GS group $(p=0.002)$.

Table III. Multivariate analysis of infectious complications in terms of associated parameters

\begin{tabular}{|lcc|}
\hline Parameter & Odds ratio* $^{*}$ & $P$-value \\
\hline Obesity** & $3.18(1.64-6.17)$ & 0.001 \\
\hline Diabetes mellitus & $0.85(0.43-1.66)$ & 0.635 \\
\hline Hypertension & $0.52(0.20-0.95)$ & 0.232 \\
\hline COPD & $1.44(1.06-3.04)$ & 0.037 \\
\hline EuroSCORE & $3.22(1.11-7.44)$ & $<0.001$ \\
\hline $\begin{array}{l}\text { Operations under } \\
\text { emergent conditions }\end{array}$ & $2.16(1.04-5.53)$ & 0.003 \\
\hline
\end{tabular}

COPD - chronic obstructive pulmonary disease, ${ }^{*} 95 \%$ confidence interval, ${ }^{* *} B M I<30 \mathrm{~kg} / \mathrm{m}^{2}$ vs. $\geq 30 \mathrm{~kg} / \mathrm{m}^{2}$, ${ }^{* * *}$ EurOSCORE $<4 \mathrm{vs} . \geq 4$.
On multivariate analysis, high EuroSCORE results $(\geq 4)$, obesity $\left(B M I>30 \mathrm{~kg} / \mathrm{m}^{2}\right)$, performing the operations under emergency situations and presence of chronic obstructive pulmonary disease were identified as significant risk factors in the development of SWI, with a respective odds ratio of 3.22, 3.18, 2.16 and 1.44 , respectively. Diabetes mellitus and hypertension were not found to significantly increase the odds ratio of SWI (Table III).

\section{Discussion}

This prospective randomized study demonstrated a significant decrease in SWIs and SSWIS after GF application at the surgical site. On the other hand, GS was associated with a statistically significantly lower number of DSWIs. On multivariate analysis, high EuroSCORE results ( $\geq 4)$, obesity $\left(\mathrm{BMI}>30 \mathrm{~kg} / \mathrm{m}^{2}\right)$, performing the operations under emergency situations and presence of chronic obstructive pulmonary disease were identified as significant risk factors in the development of SWIs.

Due to prolonged lifespan, patients referred for cardiac surgery are older and have more comorbidities than they were a few years ago. This may lead to an increase in post-operative complications [10]. In high-risk patients, the incidence of SWIs has been estimated between $12 \%$ and $20 \%$ [11]. The SWI is associated with a significantly longer hospital stay, increased medical care costs and higher mortality rate.

Although prophylactic intravenous antibiotic protocols were shown to reduce the incidence of SWIs, further attempts aim to reduce the rates of this complication [1]. Vander Salm et al. first reported the use of topical vancomycin [12]. Despite the promising results, vancomycin application was abandoned due to wound healing problems and development of vancomycin resistance [7]. 
Starting in 1987, assuming that the greatest risk of surgical wound infection was the open surgical field, Yoshii et al. attempted a procedure of spraying local antibiotics (cefazolin and gentamicin) at the surgical field. Deep surgical site infections occurred in $0.5 \%$ of 2613 cases which were included in their study [8]. In 2005, after demonstrating that topically applied gentamicin can provide high local concentrations without causing systemic side effects [2], Friberg et al. demonstrated that placement of a gentamicin collagen sponge at the sternal wound reduced the incidence of all SWIs by $53 \%$ [13]. They also noted a greater decrease in the rates of SSWI than in deep infections. In 2015, Balkanay et al. described another method of delivering local gentamicin locally at the surgical wound site [14]. In their study, they treated surgical sponges by placing them in a normal saline solution in which gentamicin was dissolved. These sponges were placed between the sternal retractors and the sternum underneath it. Their study included 100 patients. No SWIs occurred in the treatment group compared to $6(12 \%)$ cases of SWI in the control group.

One large controlled study including 6960 patients from five institutions confirmed the findings that antibiotic spray (gentamicin and cefazolin) reduced the DSWI when compared to a control group $(0.46 \%$ vs. $1.7 \%$, respectively; $p<$ 0.0001 ) [7]. In our study, we demonstrated a similar decrease in DSWIs compared to the control group (3.9\% vs. $11.1 \%$, respectively; $p=0.013$ ). Neither of two studies which evaluated the effects of antibiotic spray reported its effects on superficial SWIs. In our study, a statistically significantly lower rate of superficial SWI in the GF group was found when compared to the control group (7.7\% vs. $9.5 \%$, respectively; $p=0.035)$. In total, we found that spraying gentamicin was associated with a statistically significant decrease in the number of DSWIs ( $11.6 \%$ vs. $20.6 \%, p=0.031)$.

Gentamicin collagen sponge has been widely evaluated by several studies over the last decade. However, controversial results were reported. Application of gentamicin collagen sponge was shown to reduce the rates of SWIs in three meta-analyses [3-5], whereas another meta-analysis failed to find such evidence [6]. In our study, we demonstrated a statistically significant decrease in SSWIs in the GF group, compared to the control group ( $2.5 \%$ vs. $9.5 \%, p=0.008)$, whereas a non-significant reduction of DSWIs was found compared to the control group $(2.5 \%$ vs. $11.1 \%$, $p=0.024)$. In total, we found that application of GF significantly reduced the number of all SWIs (5.0\% vs. $20.6 \%, p=0.002$ ).

There are some limitations to our study. First of all, our study sample was relatively small. Addi- tionally, the costs of procedures and material applications were not evaluated. Moreover, patients' quality of life was not evaluated in the postoperative period. Lastly, this study focused on the short-term results of gentamicin, and long-term outcomes should be further evaluated.

In conclusion, our study demonstrated that GF application reduced the rates of overall SWI and SSWI, whereas local GS application reduced DSWIs. To clarify this issue, further prospective randomized trials with larger patient volumes are needed.

\section{Conflict of interest}

The author declares no conflict of interest.

\section{References}

1. Kreter B, Woods M. Antibiotic prophylaxis for cardiothoracic operations. Meta-analysis of thirty years of clinical trials. J Thorac Cardiovasc Surg 1992; 104: 590-9.

2. Friberg O, Jones I, Sjöberg L, Söderquist B, Vikerfors T, Källman J. Antibiotic concentrations in serum and wound fluid after local gentamicin or intravenous dicloxacillin prophylaxis in cardiac surgery. Scand J Infect Dis 2003; 35: 251-4.

3. Kowalewski M, Pawliszak W, Zaborowska K, et al. Gentamicin-collagen sponge reduces the risk of sternal wound infections after heart surgery: meta-analysis. J Thorac Cardiovasc Surg 2015; 149: 1631-40.e1-6.

4. Mavros MN, Mitsikostas PK, Alexiou VG, Peppas G, Falagas ME. Gentamicin collagen sponges for the prevention of sternal wound infection: a meta-analysis of randomized controlled trials. J Thorac Cardiovasc Surg 2012; 144: $1235-40$.

5. Raja SG. Local application of gentamicin-containing collagen implant in the prophylaxis and treatment of surgical site infection following cardiac surgery. Int J Surg 2012; 10 Suppl 1: S15-20.

6. Creanor S, Barton A, Marchbank A. Effectiveness of a gentamicin impregnated collagen sponge on reducing sternal wound infections following cardiac surgery: a meta-analysis of randomised controlled trials. Ann R Coll Surg Engl 2012; 94: 227-31.

7. Osawa H, Yoshii S, Abraham SJ, et al. Topical spraying of cefazolin and gentamicin reduces deep sternal wound infections after heart surgery: a multicenter, large volume, retrospective study. Gen Thorac Cardiovasc Surg 2016; 64: 197-202.

8. Yoshii S, Hosaka S, Suzuki S, et al. Prevention of surgical site infection by antibiotic spraying in the operative field during cardiac surgery. Jpn J Thorac Cardiovasc Surg 2001; 49: 279-81.

9. Mangram AJ, Horan TC, Pearson ML, Silver LC, Jarvis WR. Guideline for prevention of surgical site infection. Hospital Infection Control Practices Advisory Committee. Infect Control Hosp Epidemiol 1999; 20: 250-78.

10. Horan TC, Gaynes RP, Martone WJ, Jarvis WR, Emori GT. CDC definitions of nosocomial surgical site infections. Infect Control Hosp Epidemiol 1992; 13: 606-8.

11. Franco S, Herra AM, Atehortúa M, et al. Use of steel bands in sternotomy closure: implications in high-risk cardiac surgical population. Interact Cardiovasc Thorac Surg 2009; 8: 200e5. 
12. Vander Salm TJ, Okike ON, Pasque MK, et al. Reduction of sternal infection by application of topical vancomycin. J Thorac Cardiovasc Surg 1989; 98: 618-22.

13. Friberg $O$, Svedjeholm R, Söderquist B, Granfeldt $H$, Vikerfors T, Källman J. Local gentamicin reduces sternal wound infections after cardiac surgery: a randomized controlled trial. Ann Thorac Surg 2005; 79: 153-62.

14. Balkanay OO, Goksedef D, Gode S, Kılıç Z, Omeroglu SN, Ipek $G$. Does locally administered gentamicin affect the incidence of sternal wound infections after coronary artery bypass graft surgery? Turk Gogus Kalp Dama 2015; 23: $32-8$. 